\title{
Rare Clinical Presentation in a Case of Pediatric Guillain-Barré Syndrome and Rehabilitation Outcome
}

\author{
G. Sonachand Sharma ${ }^{1}$ Anupam Gupta ${ }^{1}$ Raghavendra K. ${ }^{2}$ Navin B.P. ${ }^{1}$ \\ Meeka Khanna' Ajay Asranna² Ramesh Patil ${ }^{2}$
}

${ }^{1}$ Department of Neurological Rehabilitation, National Institute of Mental Health \& Neuro Sciences (NIMHANS), Bengaluru, Karnataka, India

2Department of Neurology, National Institute of Mental Health \& Neuro Sciences (NIMHANS), Bengaluru, Karnataka, India

\begin{abstract}
Address for correspondence Anupam Gupta, MD (PMR), Department of Neurological Rehabilitation, National Institute of Mental Health \& Neuro Sciences (NIMHANS), Bengaluru 560029, Karnataka, India (e-mail: drgupta159@yahoo.co.in).
\end{abstract}

J Neurosci Rural Pract 2021;12:435-437.

\section{Case Presentation}

A 7-year-old boy with normal birth and development with no prior illness presented with acute onset symmetrical ascending pattern of the weakness of all limbs followed by restricted eye movements and drooping of both eyelids of 15 days duration. On evaluation at admission in neurology department, the child was alert and conscious with stable vitals. Cranial nerve examination showed asymmetrical bilateral partial ptosis with slight evidence of fatigability with gross global restriction of eyeball movement. Pupillary reactions and fundus examination were normal but had evidence of asymmetrical lower motor facial weakness in the form of inability to close the eyes to bury the eyelashes as well as left-sided facial deviation on attempting to smile. No evidence of bulbar weakness was noted. Motor examination showed asymmetrical flaccid

DOI https://doi.org/ 10.1055/s-0041-1727299 ISSN 0976-3147 weakness with depressed deep tendon reflex, and sensory examination was within normal limits. There was no history of fever, trauma, recent vaccination, seizure, altered consciousness, dysphagia, bladder, and bowel disturbances. The possibility of neuromuscular junction disorder versus Miller Fisher variant of Guillain-Barré syndrome (GBS) was considered as diagnosis. His electrophysiological study showed reduced compound motor action potential amplitude, normal sensory nerve action potentials with motor and sensory conduction being within normal limits. The repetitive nerve stimulation did not show evidence of neuromuscular junction disorder. Further, the cerebrospinal fluid showed a mild increase in protein with normal cytology. Screening for the antiganglioside antibody including the serum anti-GQ $1 \mathrm{~b}$ antibody was negative. He was treated with 5 cycles of small volume plasmapheresis (SVPP) followed by intravenous immunoglobulin 
( $0.4 \mathrm{~g} / \mathrm{kg}$ body weight for 5 days) given inadequate response with SVPP and referred for further inpatient rehabilitation.

At admission in the rehabilitation unit, he was bed-bound, dependent on almost all the daily activities on caregivers. The single breath count was 20 with chest expansion of $2.5 \mathrm{~cm}$ at the level of nipple (anterior axillary fold). His cranial nerves examination findings were unchanged (-Fig. 1). Upper limb motor power examination showed Medical Research Council (MRC) scale of $0 / 5$ in shoulder joint muscles, $3 / 5$ in elbow flexors, $2 / 5$ in elbow extensors, $2 / 5$ in wrist, and finger flexors of both sides with wrist and finger extensors had $1 / 5$ motor power. In the lower limbs, motor power examination showed MRC scale of $1 / 5$ in hip and knee muscles bilaterally and $3 / 5$ in ankle and toes muscles bilaterally. Bilateral hand grips were poor. The patient had poor static and dynamic trunk control.

Rehabilitation was focused with the goal to prevent complications and maximize functional ability. Physiotherapy was focused on active/active assistive range of motion exercises of all limb joints, stretching and strengthening exercises of orofacial, limb, trunk, respiratory, and extraocular muscles. For respiratory muscles training, deep breathing exercise and incentive spirometry were included. Occupational therapy was mainly focused on bed mobility, upper limb mobility, functional abilities training, hand function activities, age-appropriate activities of daily living training, and modifications. Eye care was done with night patching and four hourly applications of artificial tear drops.

After 3 weeks inpatient rehabilitation, patient developed good sitting balance (both static and dynamic). His motor power (MRC Scale) became 2/5 in shoulder groups of muscles; $3 / 5$ in elbow flexor and extensor; $3 / 5$ in flexors of wrist and finger; $2 / 5$ in wrist and fingers muscles on both sides. Lower limb muscles motor power improved to $2 / 5$ in hip muscles, $3 / 5$ in knee muscles, $4 / 5$ in ankle muscles, and $3 / 5$ in toes muscles in both sides. He became moderately independent in his daily activities including independent ambulation. He had significant recovery in extraocular movements with no ptosis ( - Fig. 2). At the time of admission, patient had severe disability with Barthel Index score of 20/100. ${ }^{1}$ Scores improved to $60 / 100$ showing significant functional recovery $(p<0.001)$.

\section{Discussion}

GBS is one of the common causes of acute flaccid paralysis in children with reported incidence of 1 to

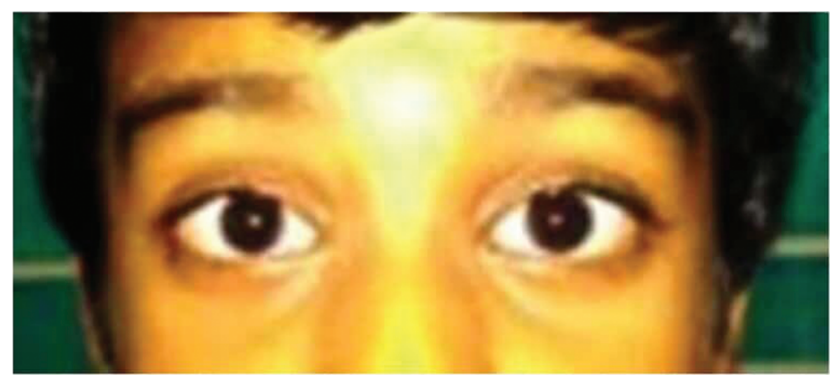

Fig. 1 Patient with complete ophthalmoplegia with recovering ptosis.
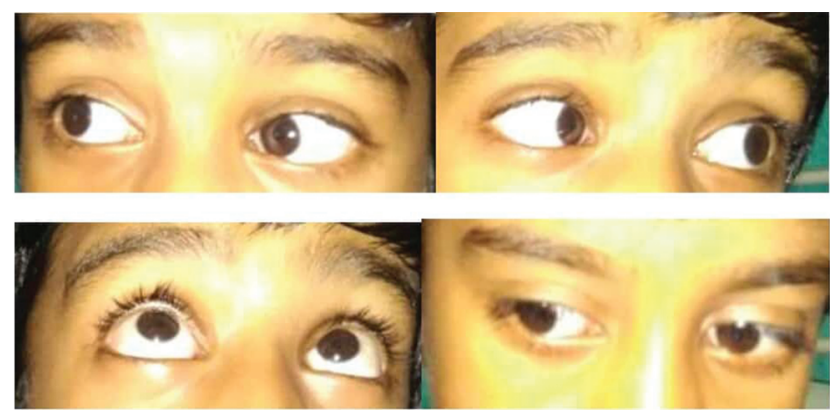

Fig. 2 Patient with recovering ophthalmoplegia at the time of discharge.

2/100,000 population..$^{2-4}$ Ocular muscle involvement or ophthalmoplegia occurs in nearly $10 \%$ of GBS patients. ${ }^{5}$ The spectrum of ophthalmic involvement in GBS is wide and can mimic central ophthalmoplegic phenomena like internuclear ophthalmoplegia and Parinaud's syndrome as well as internal ophthalmoplegia with pupillary involvement. Presence of high titers of anti-GQ1b immunoglobulin $\mathrm{G}$ antibodies usually correlate with the extent of ophthalmoplegia in GBS but can be negative in up to $15 \%$ of patients, as in our case. Since our patient had severe limbs weakness and evidence of early respiratory compromise, appropriate treatment was initiated and escalated accordingly. The improvement in the ophthalmologic deficits was spontaneous or therapy-related would be difficult comment upon.

GBS and myasthenia gravis (MG) occurring concomitantly in the same patient have been reported in the literature. ${ }^{6}$ In this case, we did not find any clinical or laboratory evidence for MG. One of the neuromuscular junction disorders, such as botulism, can also cause generalized paralysis and ptosis. ${ }^{7}$ In our patient, normal pupillary function with ascending paralysis led to the exclusion of botulism. The anti-GQ1b antibody has been found in GBS with ophthalmoplegia and MFS variants. ${ }^{8}$ Absence of ataxia and seronegative anti-GQ1b antibody excluded MFS variants in our case. Tetraplegia with seronegative anti-GQ1b antibody and multiple cranial nerves palsy manifesting as complete ophthalmoplegia, ptosis, facial weakness makes the diagnosis of GBS with a rare variant in the pediatric age group. Hence, we are adding to the literature regarding anti-GQ1b antibody-negative neuro-ophthalmological involvement of MFS spectrum, which needed prompt suspicion to initiate early treatment.

\section{Conclusion}

The GBS presenting with tetraplegia, facial palsy, and ophthalmoplegia with ptosis and seronegative anti-GQ1b antibody is a rare presentation in the pediatric population. As with other GBS, this patient showed remarkable functional and motor recovery with early and timely inpatient rehabilitation.

\section{Funding}

None. 


\section{Conflict of Interest}

None declared.

\section{References}

1 Mahoney FI, Barthel DW. Functional evaluation; The Barthel Index. Md State Med J 1965;14:61-65

2 van Doorn PA, Ruts L, Jacobs BC. Clinical features, pathogenesis, and treatment of Guillain-Barré syndrome. Lancet Neurol 2008;7(10):939-950

3 McGrogan A, Madle GC, Seaman HE, de Vries CS. The epidemiology of Guillain-Barré syndrome worldwide. A systematic literature review. Neuroepidemiology 2009;32(2):150-163

4 Levin $\mathrm{KH}$. Variants and mimics of Guillain Barré syndrome. Neurologist 2004;10(2):61-74
5 Gurwood AS, Drake J. Guillain-Barré syndrome. Optometry 2006;77(11):540-546

6 Kizilay F, Ryan HF Jr, Oh SJ. Myasthenia gravis and Guillain-Barré syndrome occurring simultaneously in the same patient. Muscle Nerve 2008;37(4):544-546

7 de Entrambasaguas M, López-Bernabé R, López-Alemany M. [Ocular myasthenia gravis: diagnostic aspects and evolution]. Rev Neurol 2007;44(7):397-403

8 Odaka M, Yuki N, Hirata K. Anti-GQ1b IgG antibody syndrome: clinical and immunological range. J Neurol Neurosurg Psychiatry 2001;70(1):50-55 